Journal of Computer Science 5 (4):323-329, 2009

ISSN 1549-3636

(C) 2009 Science Publications

\title{
Generic Algorithm with Varying Block Sizes to Improve the Capacity of a Wireless Communications Networks
}

\author{
Mahmoud Abdellaoui \\ High Institute of Electronics and Communications-Sfax, Tunisia
}

\begin{abstract}
Problem statement: Channel capacity can be an essential parameter for wireless communication to improve the link quality. Until now, the channel capacity has been evaluated by utilizing Shannon capacity theory, but it presented many limitations and restrictions. The objectives of this study were to: (i) Expose, quantify, present and to give a clear and concise exposition of the mathematical principles of the proposed generic algorithm and (ii) Calculate and simulate the SNR, the BER and the channel capacity for wireless underwater transceiver and compare with the literature references. Approach: Contrary to the existing theory (Shannon capacity theory that required and needed of the exact channel state distribution and particularly the precisely estimation of cut-off threshold profile, so, the bad estimation allowed suspending transmission even for good channel conditions), the proposed generic algorithm was used to determine the channel capacity performance with varying block size input packet information ( $\mathrm{N}$ was varied from 50-200) as a function of the optimum transmit power (from 3.5-7.0 w). This approach did not require the exact channel state distribution; but the channel was well modeled and the cut-off threshold profile had to be numerically solved. The iteration results of this algorithm were utilized to calculate and simulate the SNR, the BER and the channel capacity for wireless underwater transceiver performance. Results: The consultation performance of channel capacity wireless underwater communication, obtained with present approach, were significantly better in any transmission conditions (no suspending transmission) because the proposed algorithm was well suited to obtain results about channel capacity. In the other hand, the amount of transmitted data per resource depended on the channel conditions was performed. Blocking is no longer critical if data traffic without or with sensitivity has to be served. Data rate results were allowed of the users to be satisfied because of the wanted to achieve a certain guarantees. This was attributed to higher SNR, a lower BER and an optimal channel capacity value with the minimal transmission power. Conclusion: These results indicated that using this generic algorithm can enhance and optimize the link quality and the performance of wireless underwater communication.
\end{abstract}

Keywords: Generic algorithm, block size, packet information, capacity, channel, model

\section{INTRODUCTION}

In wireless communication and particularly in wireless underwater communication, mobile radio links usually exhibit severe multipath fading that yields serious degradation in the link-to-noise ratio (SNR) and consequently leads to higher Bit Error Rate (BER). Fading compensations like increasing link budget margin or interleaving with channel coding are typically required to improve link quality; however, these techniques are designed relative to the worst-case channel conditions, which results in low channel utilization. Transmission power, determined with the proposed algorithm, has been proven to be an efficient approach to improve the link quality and increase the channel capacity. Shannon capacity of a channel defines its maximal possible rate of data transmission for an arbitrarily small BER without any delay or complexity constraints. Many studies ${ }^{[1,2]}$ are elaborated to optimize the Shannon capacity with perfect channel side information at both the transmitter and receiver. Essential disadvantages for this approach concern the transmitter power and rate according to the channel state: allocate higher power levels and rates for good channel conditions, lower power levels and rates for unfavorable channel conditions and suspend transmission when the SNR is lower than a cut-off threshold. In this study, we propose an algorithm scheme with varying block sizes which does not require the exact channel state distribution; but, the channel is well modeled, the cut-off threshold has to be numerically solved. The iteration results of this algorithm are utilized to calculate and simulate the SNR and the BER for Wireless underwater transceiver 
performance. Numerical examples based on mathematical analysis are provided to show the effectiveness of the proposed algorithm. And to appear that the generic algorithm is robustness, simples, accurate and is therefore utilized to extract easily the crucial parameters required, especially, for the underwater communication system: like the link Signal to Noise Ratio (SNR) and the higher Bit Error Rate (BER).

\section{MATERIALS AND METHODS}

In this study, we describe a generic algorithm utilized to determine the channel capacity, SNR, $\mathrm{BER}, \ldots$ of the wireless underwater communication. The proposed algorithm based on the varying size of the transmission information data was detailed as method of research and to respond to: How did precisely determine about solving or making progress on the problem. This provided sufficient detail to allow the work to be repeated by others.

Mathematical analysis: In wireless communications, transmission power is efficient approach to improve network capacity. Let SNR denote the signal-to noise ratio and $\mathrm{P}(\mathrm{SNR}(\mathrm{x}))$ be the transmit power, which depends to an average power constraint:

$\int_{0}^{\infty} \mathrm{P}(\operatorname{SNR}(\mathrm{x})) \mathrm{p}(\Theta) \mathrm{d}(\Theta) \leq \overline{\mathrm{P}}$

Where:

$\mathrm{p}(\Theta)=$ The probability density function of SNR

$\overline{\mathrm{P}}=\mathrm{A}$ constant

Then the optimal transmission power is:

$\mathrm{P}(\operatorname{SNR}(x))=\left\{\begin{array}{l}\overline{\mathrm{P}}\left(\frac{1}{\operatorname{SNR}(x)}-\frac{1}{\overline{\operatorname{SNR}(x)}}\right) \\ 0\end{array}\right.$

Where, $\overline{\operatorname{SNR}(x)}$ is the cutoff threshold that satisfies the following equation:

$\int_{\operatorname{SNR}(x)}^{\infty}\left(\frac{1}{\overline{\operatorname{SNR}(x)}}-\frac{1}{\operatorname{SNR}(x)}\right) \mathrm{p}(\Theta) \mathrm{d}(\Theta)=1$

The channel capacity is given by:

$\mathrm{B} \int^{\infty} \log _{2}(\mathrm{SNR}(\mathrm{x}) / \overline{\operatorname{SNR}(\mathrm{x})}) \mathrm{p}(\Theta) \mathrm{d}(\Theta)$ where, B is the channel bandwidth. Note that the optimal transmit power depends only on $\overline{\operatorname{SNR}(x)}$, once $\overline{\operatorname{SNR}(x)}$ is defined, the transmit power is determined. Even, the channel state can be perfectly estimated by the transmitter, the exact distribution of SNR has to be estimated. A possible way to determine the power when you can to estimate the probability distribution of SNR and then solve (3) to get $\overline{\operatorname{SNR}(x)}$ (this parameter has to be numerically solved). Many property of the cutoff threshold are deducted, determined and given from the following study for these cases:

Let $\mathrm{X} \geq 0$ be a random variable denoting generic SNR, satisfying $E\left[1 / \mathrm{X}^{2}\right] \leq \infty$ :

$\operatorname{DefineG}(\bar{\rho})=\int_{\bar{\rho}}^{\infty}\left(\frac{1}{\rho}-\frac{1}{\bar{\rho}}\right) \mathrm{p}(\Theta) \mathrm{d}(\Theta)-1$

Where:

$\rho=\operatorname{SNR}(x)$

$\bar{\rho}=\overline{\operatorname{SNR}(x)}$

Then $\mathrm{G}(\overline{\operatorname{SNR}(\mathrm{x})})$ is decreasing in $\overline{\operatorname{SNR}(\mathrm{x})}$ and Eq. 3 has a unique zero point which lies in $(0,1)$. Noting that:

$$
\begin{aligned}
\mathrm{G}(\overline{\operatorname{SNR}(x)})= & \frac{1-\mathrm{F}(\overline{\operatorname{SNR}(x)}}{\overline{\operatorname{SNR}(x)}} \\
& -\int_{\overline{\operatorname{SNR}(x)}}^{\infty} \frac{1}{\operatorname{SNR}(x)} \mathrm{p}(\Theta) \mathrm{d}(\Theta)-1
\end{aligned}
$$

Where:

$\mathrm{G}=$ Continuously differentiable

$\mathrm{F}=$ Determined with direct computation gives by:

$\mathrm{G}^{\prime}\left(\overline{\operatorname{SNR}(\mathrm{x})}=-\frac{1-\mathrm{F}(\operatorname{SNR}(\mathrm{x}))}{[\operatorname{SNR}(\mathrm{x})]^{2}} \leq 0\right.$

Implying $\mathrm{G}(\mathrm{SNR}(\mathrm{x}))$ is strictly decreasing in SNR(x). Evidently:

$\underline{\lim _{0}} \mathrm{G}(\operatorname{SNR}(\mathrm{x}))=\infty, \underline{\lim _{\infty}} \mathrm{G}(\operatorname{SNR}(\mathrm{x}))=0$

Which combined with (6) implies (5) has a unique solution. Obviously:

$\mathrm{G}(1)=-\left[\mathrm{F}(1)+\int_{1}^{\infty} \frac{1}{\mathrm{G}(\operatorname{SNR}(\mathrm{x}))} \mathrm{p}(\Theta) \mathrm{d}(\Theta)\right] \leq 0$

Which combining with (7) implies the solution of (5) lies in $(0,1)$. Noting that: 
$\mathrm{G}(\overline{\operatorname{SNR}(\mathrm{x}))}$

$=E\left[\left(\frac{1}{\overline{G(\operatorname{SNR}(x))}}-\frac{1}{\operatorname{G(SNR}(x))}\right) I_{\left[X \leq \frac{1}{\overline{G(\operatorname{SNR}(x))}}\right]}\right]-1$

where, $I_{[.]}$is the indicator function, the solution of $\mathrm{G}(\overline{\operatorname{SNR}(\mathrm{x})})=0$ can be solved by stochastic approximation. For this purpose, we define a sequence of step sizes. Denote $a_{K}=1 / K^{\gamma}, K \geq 1, \gamma \in(0.25,1)$, which satisfy:

$\sum_{\mathrm{K}=1}^{\infty} \mathrm{a}_{\mathrm{K}}=\infty, \sum_{\mathrm{K}=1}^{\infty} \mathrm{a}_{\mathrm{K}}^{2} \leq \infty$

To design the optimal transmission power scheme with varying block sizes, we choose to update the estimation of $\mathrm{G}(\overline{\mathrm{SNR}(\mathrm{x})})$ block by block with each block consisting of $\mathrm{N}$ symbols whose varies from 1 to 200. Let $X_{k}(L)$ denote the channel state of the L-th symbol of the $\mathrm{k}$-th block and $\mathrm{Z}_{\mathrm{k}}=\left(\mathrm{X}_{\mathrm{k}}(1)\right.$, $\left.\mathrm{X}_{\mathrm{k}}(2), \ldots, \mathrm{X}_{\mathrm{k}}(\mathrm{N})\right)^{\mathrm{T}}$. Then, the parameter $\overline{\operatorname{SNR}(\mathrm{x})}$ is update as follows:

$\operatorname{SNR}(x)_{k+1}=\left\{\begin{array}{c}\operatorname{SNR}(x)_{k}+a_{k} T_{N}\left(\operatorname{SNR}(x), Z_{k+1}\right) \\ \operatorname{SNR}(x)\end{array}\right.$

With $\operatorname{SNR}(\mathrm{x})_{\mathrm{k}}+\mathrm{a}_{\mathrm{k}} \mathrm{T}_{\mathrm{N}}\left(\operatorname{SNR}(\mathrm{x})_{\mathrm{k}}, \mathrm{Z}_{\mathrm{k}+1}\right) \in(0,1)$; where:

$\mathrm{T}_{\mathrm{N}}()=.\frac{1}{\mathrm{~N}} \sum_{\mathrm{L}=1}^{\mathrm{N}}\left[\frac{1}{\operatorname{SNR}(\mathrm{x})}\right)-\left(\frac{1}{\mathrm{X}_{\mathrm{k}+1}(\mathrm{~L})}\right] \mathrm{I}_{\left[\mathrm{X}_{\mathrm{k}+1}(\mathrm{~L}) \geq \operatorname{SNR}(\mathrm{x})\right]}-1$

Convergence and efficiency: Equation 11 is a standard stochastic approximation algorithm, whose convergence can be given by adapting the next study with $^{[3,4]}$. In the other hand, if assume the channel state's stationary and ergodic, as:

$\varliminf_{\infty} \rho_{k}=\bar{\rho}_{k}$

Note that (11) can be written as:

$\left\{\begin{array}{c}\rho_{k+1}=\rho_{k}+a_{k} y_{k+1} \\ y_{k}=G\left(\rho_{k}\right)+\varepsilon_{k}\end{array}\right.$

Where:

$\rho_{\mathrm{k}}=\operatorname{SNR}(\mathrm{x})_{\mathrm{k}}$

$\varepsilon_{\mathrm{k}+1}=\stackrel{\Delta}{\longleftrightarrow} \varepsilon_{\mathrm{k}+1}\left(\rho_{\mathrm{k}}, \mathrm{Z}_{\mathrm{k}+1}\right)-\mathrm{G}\left(\rho_{\mathrm{k}}\right)$

Let $\mathrm{F}_{\mathrm{k}}=\sigma\left(\mathrm{Z}_{1}, \mathrm{Z}_{2}, \ldots, \mathrm{Z}_{\mathrm{k}}\right)$. Since $\mathrm{E}\left[_{\mathrm{TN}}\left(\rho_{\mathrm{k}}, \mathrm{Z}_{\mathrm{k}+1}\right) \mid\right.$ $\left.F_{k}\right]=G\left(\rho_{k}\right),\left\{\left(\varepsilon_{k}, F_{k}\right), k \geq 1\right\}$ is a martingale difference sequence with finite second order moment. To prove the study, it suffices to verify the conditions of ${ }^{[3]}$. Verification is straightforward and compared with results of ${ }^{[5]}$. Note that, for any given $\rho$ :

$\mathrm{E}\left[\varepsilon_{\mathrm{k}+1}^{2}\left(\rho_{\mathrm{k}}, \mathrm{Z}_{\mathrm{k}+1}\right) \mid \mathrm{F}_{\mathrm{k}}\right] \leq \mathrm{N} / \rho^{2}+\mathrm{NE}\left(1 / \mathrm{X}^{2}\right)<\infty$

This is bounded for any $\mathrm{k}$.

In the other hand, if assume the channel state as stationary and ergodic (same $E\left[1 / X^{\mathfrak{\eta}}\right]<\infty$ for some $\mathrm{n}>2$ ). Let $G^{\prime}\left((\bar{\rho})=-\frac{1-F(\bar{\rho})}{\rho^{2}}\right.$. Then, we have:

$\frac{\rho_{\mathrm{k}}-\overline{\rho_{\mathrm{k}}}}{\sqrt{\mathrm{a}_{\mathrm{k}}}} \stackrel{\mathrm{d}}{\longrightarrow} \mathrm{N}\left(0, \mathrm{~S}_{0}\right)$

Where:

$S_{0}=\frac{\left.\operatorname{var}\left[\left(\frac{1}{\overline{\rho_{k}}}-\frac{1}{X}\right)\right] I_{[X \geq \overline{\rho]}}\right)}{N\left(-G^{\prime}(\overline{\rho)})\right.}$

When $\rho_{\mathrm{k}} \rightarrow \overline{\rho_{\mathrm{k}}}$ with probability one, we can obtain:

$\operatorname{limE}\left[\varepsilon_{k}^{2} \mid F_{k}\right]=E\left[T_{N}\left(\rho_{k}, Z_{k}\right)-G(\rho)\right]^{2}=\operatorname{var}\left(\left[\frac{1}{\overline{\rho_{k}}}-\frac{1}{X}\right] I_{[X \geq \rho]}\right) / N$

Moreover, using the Holder's inequality and the assumption:

$\lim _{\infty} \sup _{\mathrm{k}} \mathrm{E}\left[\varepsilon_{\mathrm{k}}^{2} \mathrm{I}_{[|\varepsilon \mathrm{k}| \geq \mathrm{T}]}\right]=0, \forall \mathrm{T}>0$

In the algorithm (11), the parameter $\mathrm{N}$ varies from $1-200$. The smaller $N(1 \leq N \leq 200)$, the more frequently the cut off threshold is update, but in this case $\operatorname{SNR}(x)_{k}$ can be variant along iterations; on the other hand, for larger $\mathrm{N}(20<\mathrm{N} \leq 200)$, the cut off threshold is less frequently updated, but the $\operatorname{SNR}(\mathrm{x})_{\mathrm{k}}$ has smaller variances.

\section{RESULTS}

To show the effectiveness of the proposed algorithm with varying block sizes, let us give some application results in terms of the wireless underwater communication performance like SNR, BER and channel capacity with an optimal transmission power for different modulations and codes. Recall to: In wireless communication and particularly in wireless underwater communication ${ }^{[6]}$, mobile radio links 
usually exhibit severe multipath fading that yields serious degradation in the link signal-to- noise ratio and consequently leads to higher bit error rate. The multipath fading environment can be characterized by a variety of models (Rayleigh-L-model, Nokagami model ... $)^{[7-9]}$. In the other hand, with the object to summarize the major results in relation to any hypotheses advanced in this study and obtained based on the generic algorithm, we presented, in the following subsections, a higher SNR, a lower BER and an optimal channel capacity with minimum transmit power.

Transmission power: To solve the optimal $\overline{\operatorname{SNR}(x)}$, $\mathrm{G}\left(\operatorname{SNR}(\mathrm{x})_{\mathrm{k}}\right)$ is evaluated by numerical integration and the optimal $\overline{\operatorname{SNR}(\mathrm{x})_{\mathrm{k}}}$ is computed and with parameters $\mathrm{m}=0.7, \quad \mathrm{w}=0.6$, we can obtain the optimal $\overline{\operatorname{SNR}(\mathrm{x})_{\mathrm{k}}}=0.42$.

The channel gain is Nakagami-m distributed ${ }^{[10,11]}$ and the received SNR has the gamma distribution expressed as:

$\mathrm{p}(\gamma, \theta, \mathrm{m})=\left(\theta^{\mathrm{m}} / \Gamma(\mathrm{m})\right) \theta^{\mathrm{m}-1} \mathrm{e}^{-\theta \gamma}$

Compared with ${ }^{[12]}$, Rayleigh distribution with a random mode $\sqrt{\mathrm{Y}}$ can be expressed as:

$\mathrm{p}(\mathrm{x} / \mathrm{Y})=(\mathrm{x} / \mathrm{Y}) \exp \left(-\mathrm{x}^{2} / 2 \mathrm{Y}\right)$

In these conditions, the transmit power required to transmit a packet of data decrease to any great extend (ratio of $10^{-3}$ ) with many frequencies, for a distance of $1 \mathrm{Km}$.

For transmitting the packet of data from emitter to receiver, the total energy received and detected by the receiver after $1 \mathrm{Km}$ is $0.1 \mathrm{~mW}$ for the emission power is $0.1 \mathrm{~W}$. Figure 1 shows the consumed power that's varying to $100 \mathrm{~mW}$ with $1 \mathrm{Kbits}^{\mathrm{sec}}{ }^{-1}$ input data rates, in which emitter and receiver are arranged linearly and are separated at distance $\mathrm{d}=1 \mathrm{Km}$ for the depth of approximately $100 \mathrm{~m}$.

Signal-to-Noise Ratio (SNR): In the wireless underwater communication, the bandwidth will likely be much higher than the data rate, so that the system can operate at very low Signal to Noise Ratio (SNR). This means that a wireless underwater communication network will be able to achieve high data rates with relatively low transmit power. A key point is that: In this regime, the capacity increases almost linearly with power, whereas in the bandwidth limited regime (high SNR), capacity increases only as the logarithm of signal power (which means that a linear increases in data rate requires exponentially more power).

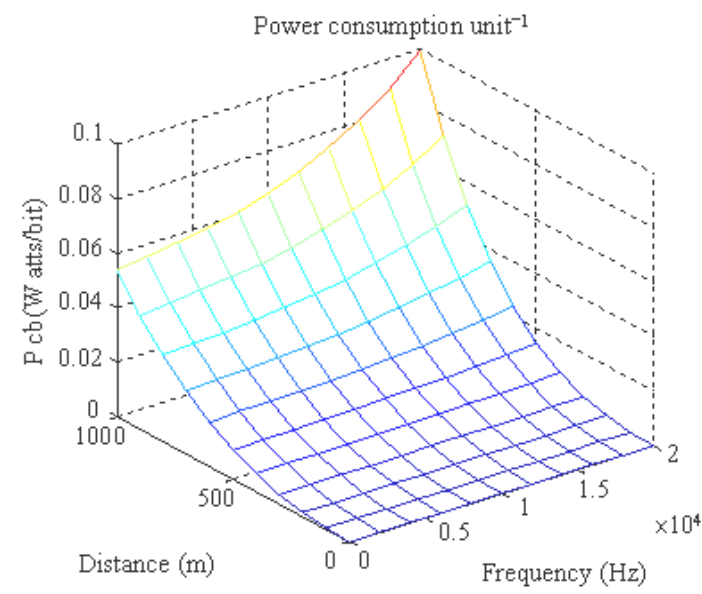

Fig. 1: Consumed power with transmission distance and frequency

Consider Rayleigh fading channel on AWGN channel, with parameters $\mu=1.75, \sigma=0.4$. Running the proposed algorithm based on varying block sizes with $\mathrm{N}=5$ and $\mathrm{N}=200$ yields $\overline{\operatorname{SNR}(\mathrm{x})} \mathrm{k}=0.75$; showing that when $\mathrm{N}=200, \mathrm{SNR}(\mathrm{x})_{\mathrm{k}}$ converges to the optimal value very quickly. The background noise of the system is fixed through out the simulations. Under, the simulation results of relative signal to Noise Ratio versus frequency. The SNR's varying between 70 and $50 \mathrm{~dB}$ from a maximum distance of $10 \mathrm{Km}$, with several frequencies from $1-20 \mathrm{KHz}$ that's in completely harmony and in a good agreement with Stojanovic simulation results ${ }^{[13]}$. As for that, the noise level is calculated to achieve $70 \mathrm{~dB}$ SNR at maximum range. This SNR level ensures about $10^{-3}$ Bit Error Rate on AWGN channel and multi-path Rayleigh fading channel. To evaluate the impact on SNR and in the other way, we can determine the symbol error rate and then, we deduct the SNR. The relation between $\mathrm{E}_{\mathrm{s}} / \mathrm{N}_{0}$ and SNR is:

$$
\begin{aligned}
& \left(\frac{\mathrm{E}_{\mathrm{s}}}{\mathrm{N}_{0}}\right)_{\mathrm{dB}}=\left(\frac{\mathrm{E}_{\mathrm{b}}}{\mathrm{N}_{0}}\right)_{\mathrm{dB}}+10 \log \mathrm{k} \\
& =\mathrm{SNR}_{\mathrm{dB}}-10 \log \left(\frac{\mathrm{R}}{\mathrm{B}}\right)+10 \log \mathrm{k}
\end{aligned}
$$

Where:

$\mathrm{R}=$ the signal's bit rate

$\mathrm{B}=$ the signal's bandwidth

$\mathrm{K}=$ the number of bit per symbol

The conditional symbol error rate, compared and adapted with $^{[14]}$, is given by: 


$$
\begin{aligned}
& P_{\text {s-BPSK }}\left[\frac{E_{b}}{N_{0}} \backslash \varphi\right]=\frac{1}{2} \operatorname{erfc}\left[\sqrt{\frac{E_{b}}{N_{0}}} \cos (\varphi)\right] \\
& P_{\text {s-QPSK }}\left[\frac{E_{b}}{N_{0}} \backslash \varphi\right]=\frac{1}{4} \operatorname{erfc}\left[\begin{array}{l}
\left(\sqrt{\frac{E_{b}}{N_{0}}}(\cos (\varphi)-\sin (\varphi))\right) \\
+\left(\sqrt{\frac{E_{b}}{N_{0}}}(\cos (\varphi)+\sin (\varphi))\right)
\end{array}\right.
\end{aligned}
$$

Bit Error Rate (BER): Note that, in general case, on the performance and complexity of irregular variable length codes for near-capacity joint source (transmit power, SNR, BER...) and channel coding. In the other hand, total outage capacity of randomly-spread coded with linear multi-user receivers over multipath fading channels, for thus, in the encoder block used in this study, the HADAMARD code increases the effective receiver BER by providing additional coding gain. The coding gain, provided by the HADAMARD code, allows one or two tones to fade without significant impact on the receiver BER. The HADAMARD scheme provides a significant decrease in BER for increasing levels of SNR per bit. The BER performance results have been determined with the maximum SNR value, the signal interference and particularly $E_{b} / N_{O}$.

Figures 2-5 show a simulated BER for several modulation types as a function of $\mathrm{E}_{b} / \mathrm{N}_{\mathrm{O}}$, in which $\mathrm{E}_{\mathrm{b}}$ is average energy of a modulated bit of transmitted packet and $\mathrm{N}_{\mathrm{O}}$ is the noise power spectral density. The signal is correctly demodulated, if the $\mathrm{E}_{\mathrm{b}} / \mathrm{N}_{\mathrm{O}}$ ratio have an acceptable value higher than $15 \mathrm{~dB}^{[15]}$. It can be seen that the BER is less than $10^{-3}$ for $\mathrm{E}_{\mathrm{b}} / \mathrm{N}_{\mathrm{O}}$ once higher than $18 \mathrm{~dB}$ over the frequency range within the operating range and that's verified only in the case of QPSK with CRC coding followed to convolution encoders.

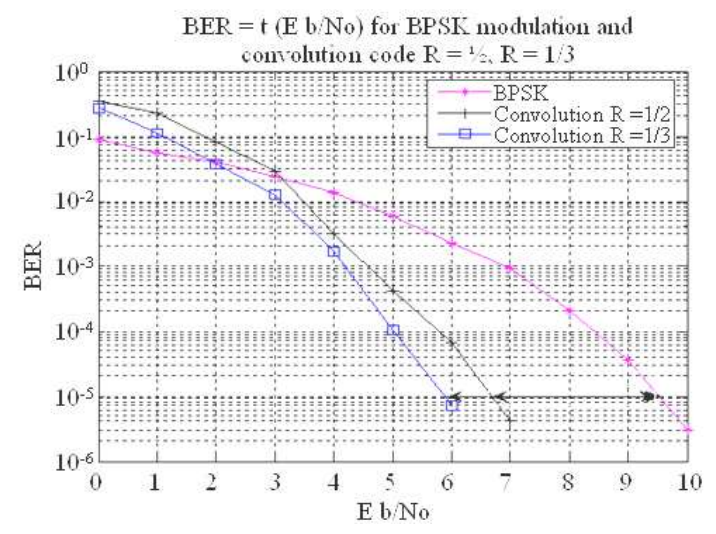

Fig. 2: BER for BPSK
Table 1 summarized the results (in terms of performance) of the wireless underwater receiver like BER and transmission power for different modulations and codes with the optimal parameters $\left(\operatorname{SNR}(\mathrm{x})_{\mathrm{k}}, \mathrm{N}\right)$ of the proposed algorithm specified by varying block sizes.

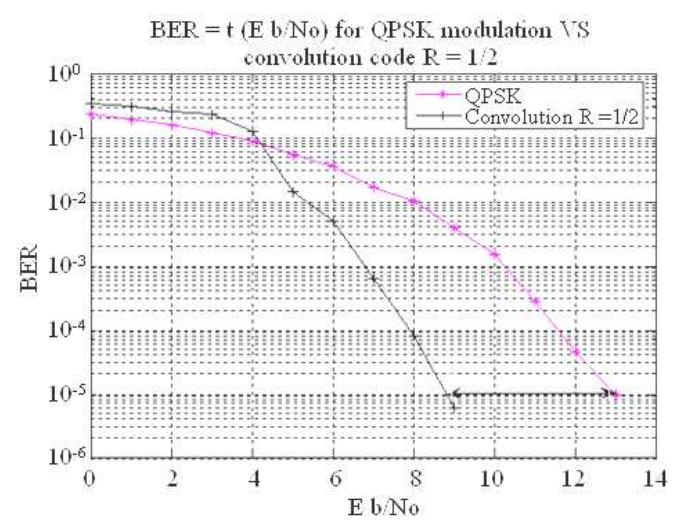

Fig. 3: BER for QPSK

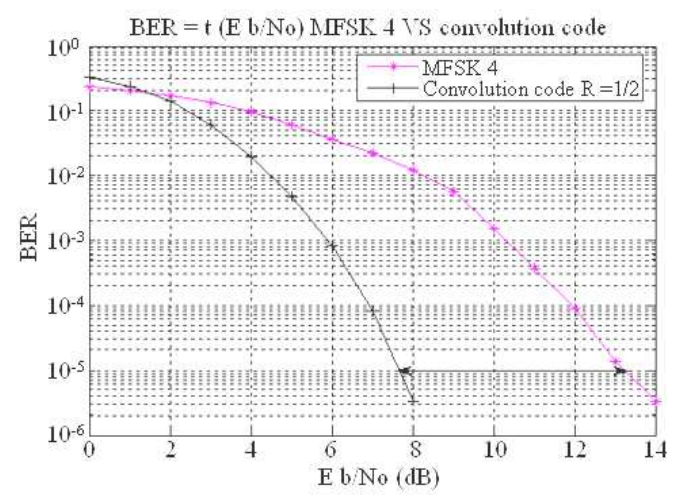

Fig. 4: BER for MFSK4

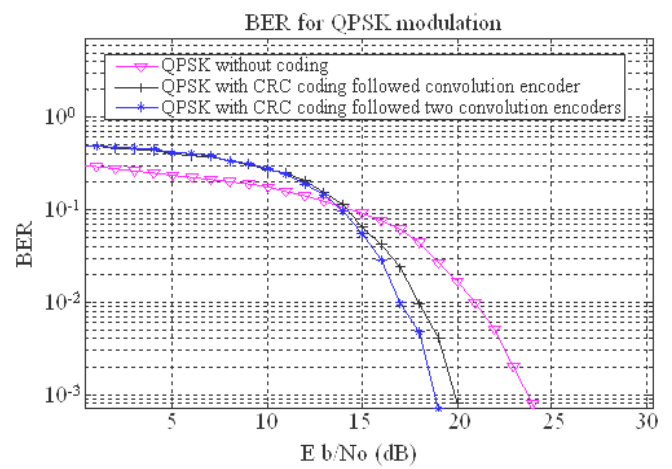

Fig. 5: BER for QPSK with many encoding 
Table 1: Wireless underwater communication performance

\begin{tabular}{lllll}
\hline $\begin{array}{l}\text { Modulation/ } \\
\text { channel gain } \\
\text { model }\end{array}$ & $\begin{array}{l}\text { Optimal } \\
\text { parameters: } \mathrm{N}, \\
\mathrm{SNR}(\mathrm{x})_{\mathrm{k}}\end{array}$ & $\begin{array}{l}\text { Coding } \\
\text { rate }\end{array}$ & BER & $\begin{array}{l}\text { Trans. } \\
\text { power } \\
(\mathrm{W})\end{array}$ \\
\hline BPSK/Nakagami & $75 ; 0.12$ & $1 / 2$ & $4.10^{-3}$ & 3.5 \\
BPSK/Rayleigh & $50 ; 0.16$ & $1 / 3$ & $4,5.10^{-3}$ & 4.1 \\
QPSK/Nakagami & $100 ; 0.42$ & $1 / 2$ & $7.10^{-3}$ & 6.0 \\
MFSK4/Rayleigh & $100 ; 0.32$ & $1 / 2$ & $6.10^{-3}$ & 5.5 \\
QPSK/Rayleigh & $200 ; 0.75$ & $3 / 4$ & $18.10^{-3}$ & 7.0 \\
\hline
\end{tabular}

\section{DISCUSSION}

Executants the proposed algorithm with varying block sizes input packet information which does not require the exact channel state distribution, but with a precise channel model allowing the cut-off threshold profile has to be numerically solved by the running algorithm iterations, all the optimal wireless underwater communication parameters are easily and accurately obtained, as channel capacity, SNR, BER,... Among the results obtainable using the generic algorithm, the SNR simulations are taken from underwater wireless communication specifications giving $\mathrm{N}$ varies from 50 200. Performance is evaluated for three different channels: AWGN channel, Nakagami channel, Rayleigh channel. Proposed method is evaluated for 4 different cases: $\mathrm{N}=50,75,100$ and 200. The generic algorithm shows the best SNR performance compared with the literature references because for average SNR values greater than $5 \mathrm{~dB}$ and performs highly sensitive to channel selectivity. It can be noticed that in the region of high values of SNR, channel estimates stop to act as deteriorating factor, but since all considered estimators depends on channel estimates, bad performance in the region of low values of SNR is expected.

Here is another result concerning BER; Examination of Fig. 2-5 shows that BER is inversely proportional to the delay spread. In the process, the BER was calculated using equations (20) and (21) and simulated with the generic algorithm. Note that the value of the BER is compared from the simulated BER's obtained from the generic algorithm and the calculated BER's from the prediction equations. It's clear, from Table 1, that the BER produces the lowest value for underwater wireless communication with QPSK modulation in AWGN-Rayleigh channel $\left(\mathrm{BER}=1,8.10^{-4}\right)$.

Finally, channel capacity has been proven to be an efficient element to improve the link quality. Our strategy or approach supposed that the capacity of varying channel equals the capacity of an ordinary memory less channel with additive Gaussian noise of power for the same input information with varying block size and at the same time by different states of the channel (a code of block length $\mathrm{N}$ comprises a set of codeword: N varies from 50-200). Indeed, the optimal transmission power in the cut-off threshold suffices to achieve some wireless link properties. On the other hand, since the Multiple Access (MAC) and intuitive method of the channel state information is unavailable, the computations are less lucid and appear to rely, to a degree, on analytical artifices. When we believe the capacity to yet remain unchanged, does not yield to such a simple artifice. On the contrary, it apparently requires more complex calculations

\section{CONCLUSION}

The transmission power is a very efficient and an important property in wireless networks. In this study, we proposed a generic algorithm specified with varying block sizes input data information. The optimal signalto-noise ratio is evaluated and determined by numerical integration and it's computed with mathematical analysis. Then, we developed some properties as: transmission power, receiver SNR, BER for the wireless underwater communication. These results are given to show that the proposed algorithm works very well and in the other hand, has indicated that using this generic algorithm can enhance and optimize the link quality and the performance of wireless underwater communication.

\section{REFERENCES}

1. Goldsmith, A.J. et al., 1997. Capacity of fading channels with channel side information. IEEE Trans. Inform. Theor., 43: 1986-1992. DOI: 10.1109/18.641562.

2. Abdellaoui, M. et al., 2006. Determination of the underwater channel characteristics to improve a multi band OFDM communication. Acad. J. Incorporat., $\quad 1$ : 431-443. http://scialert.net/qredirect.php?doi=tasr.2006.431. 443\&linkid=pdf

3. Chen, H.F. et al. 2005. Applying author ship analysis to extremist-group Web forum messages. IEEE Intell. Syst., 20: 67-75. DOI: 10.1109/MSI.2005.81

4. Fabian, V. et al., 1968. On asymptotic normality in stochastic approximation. Ann. Math. Stat., 39: 1327-1332.

http://projecteuclid.org/DPubS? service=UI\&versio $\mathrm{n}=1.0 \&$ verb=Display\&handle=euclid.aoms $/ 117769$ 8258 
5. Liu, Z. et al., 2008. Online Optimization of data transmission policies for wireless networks. IEEE Trans. Automat. Control, 53: 2633-2638. DOI: 10.1019/TQC.2008.200.7886

6. Sozer, E.M. et al., 2000. Underwater acoustic networks. IEEE J. Ocean. Eng., 25: 72-83. http://ieeexplore.ieee.org/xpl/freeabs_all.jsp?\&arnu mber $=820738$

7. Hansen, F. et al., 1977. Mobile fading-Rayleigh and lognormal superimposed. IEEE Trans. Veh. Technol., 26: 332-335.

http://ieeexplore.ieee.org/xpl/freeabs_all.jsp?arnum ber $=1622403$

8. Abdi, A. et al., 2000. Comparison of DPSK and MFSK bit error rates for $\mathrm{k}$ and Rayleigh lognormal fading distributions. IEEE Commun. Lett., 4 : 122-124. http://ieeexplore.ieee.org/xpl/tocresult.jsp?isYear= 2000\&isnumber $=18183 \&$ Submit32=View+Content

9. Proakis, J. et al., 1991. Coded modulation for digital communication over Rayleigh fading channels. IEEE J. Ocean. Eng., 16: 66-73. DOI: $10.1109 / 48.64886$

10. Suzuki, H., 1977. A statistical model for urban radio propagation. IEEE Trans. Commun., 25: 673-680. http://ieeexplore.ieee.org/xpl/tocresult.jsp?is Year= 1977\&isnumber $=23892 \&$ Submit32=Go+To+Issue

11. Avidor, D. et al., 2008. Transmit power distribution of Wireless Ad-hoc networks with topology control. IEEE Trans. Wireless Commun., 7: 1111-1116

http://ieeexplore.ieee.org/Xplore/login.jsp?url=/iel5 /7693/4489728/04489731.pdf?temp=x
12. Kalofonos, D.N. et al., 2003. Performance of adaptive MC-CDMA detectors in rapidly fading Rayleigh channels. IEEE Trans. Wireless Commun., 2: 229-239. DOI: 10.1109/TWC.2003.808960

13. Stojanovic, M. et al., 1995. Multisensor multiuser receivers for time dispersive multipath fading channels. Proceedings of the IEEE International Symposium on Information Theory, Sept. 17-22, IEEE Xplore Press, USA., pp: 332. DOI: 10.1109/ISIT.1995.550369

14. Chen, S. et al., 2008. Adaptive minimum symbol error rate beam forming assisted detection for quadrature amplitude modulation. IEEE Trans. Wireless Commun., 7: 1140-1145. DOI: 10.1109/TWC.2007.060840

15. Scussel, K.E. et al., 1997. A new MFSK acoustic modem for operation in adverse underwater channels. Proceeding of the IEEE Conference on Oceans, Oct. 6-9, IEEE Xplore Press, Washington DC., USA., $\quad$ pp: 247-254. http://ieeexplore.ieee.org/Xplore/login.jsp?url=/iel3 /4919/13761/00634370.pdf?temp=x 